\title{
Vimentin as a potential therapeutic target in sorafenib resistant HepG2, a HCC model cell line
}

\author{
Ankita Makol', Harpreet Kaur', Sakshi Sharma', Shruthi Kanthaje', Ramanpreet Kaur², and Anuradha Chakraborti' \\ Departments of ${ }^{1}$ Experimental Medicine and Biotechnology and ${ }^{2}$ Hepatology, Postgraduate Institute of Medical Education \& Research, \\ Chandigarh, India
}

Background/Aims: Hepatocellular carcinoma (HCC) is the most common liver cancer with high mortality rate in patients suffering from liver diseases. The drug of choice used in advanced-stage of HCC is sorafenib. However, adaptive resistance has been observed in HCC patients undergoing long-term sorafenib treatment, lowering its effectiveness. Hence, it is important to overcome drug resistance to improve overall management of HCC. Here, we have identified a candidate biomarker for sorafenib resistance in a HCC model cell line, HepG2.

Methods: Initially, comparative proteomic profiling of parental HepG2 [HepG2 (P)] and sorafenib-resistant HepG2 [HepG2 (R)] cells was performed via MALDI (matrix-assisted laser desorption/ionization) which revealed the deregulation of vimentin in HepG2 (R) cells. Gene and protein level expression of vimentin was also observed through quantitative real-time polymerase chain reaction (qRT PCR) and fluorescence-activated cell sorting (FACS), respectively. Furthermore, withaferin $A$ was used to study regulation of vimentin expression and its significance in sorafenib resistance.

Results: Both gene and protein level of vimentin expression was found to be downregulated in HepG2 (R) in comparison to HepG2 (P). Interestingly, the study demonstrated that withaferin A further lowered the expression of vimentin in HepG2 (R) cells in a dose-dependent manner. Also, inhibition of vimentin lowered $A B C G 2$ expression and decreased cell viability in parental as well as sorafenib resistant HepG2 cells.

Conclusions: Hence, our study for the first time highlighted the probable therapeutic potential of vimentin in sorafenib resistant HepG2, a HCC model cell line. (Clin Mol Hepatol 2020;26:45-53)

Keywords: Carcinoma, Hepatocellular; Sorafenib resistance; Vimentin; Withaferin A

\footnotetext{
Study Highlights

The present study highlights the significance of vimentin as potential therapeutic target as its inhibition with withaferin A results to overcome sorafenib resistance as well as reduces cancer cells viability.
}

\begin{abstract}
Abbreviations:
conc., concentrations; DMSO, dimethyl sulfoxide; EMT, epithelial-mesenchymal transition; FBS, fetal bovine serum; GAPDH, glyceraldehyde 3-phosphate dehydrogenase; GRP78, glucose regulated protein 78; HCC, hepatocellular carcinoma; HepG2 (P), HepG2 parental; HepG2 (R), HepG2 sorafenib resistant; IC, inhibitory concentration; MALDI, matrix-assisted laser desorption/ionization; MEM, minimum essential media; PBS, phosphate-buffered saline; PMF, peptide mass fingerprinting; qRT PCR, quantitative real-time polymerase chain reaction; SDS-PAGE, sodium dodecyl sulfate polyacrylamide gel electrophoresis; TOF, time of flight
\end{abstract}

Corresponding author : Anuradha Chakraborti

Department of Experimental Medicine and Biotechnology, Postgraduate Institute of Medical Education \& Research, Research Block B, Chandigarh 160012 , India

Tel: +91-172-275-5230

E-mail: superoxide14@gmail.com

https://orcid.org/0000-0001-8306-7558 


\section{INTRODUCTION}

Hepatocellular carcinoma (HCC) is the second leading cause of cancer-related deaths globally. HCC, the primary liver cancer, is regarded as the third most common malignancy worldwide. ${ }^{1,2}$ The major risk factors of HCC includes hepatitis C and B viral infections, fungal metabolite aflatoxin $\mathrm{A} 1$ exposure, alcohol intake, obesity, and non-alcoholic fatty liver diseases. ${ }^{3,4} \mathrm{HCC}$ is generally asymptomatic in nature and hence, is mainly diagnosed in the advanced stage. ${ }^{5}$ The lack of primary screening leads to an increased mortality to incidence ratio. Furthermore, sorafenib, an orally active multikinase inhibitor, is the first-line of therapy showing a significant survival benefit in patients with advanced HCC. Sorafenib has a direct effect on the components of tyrosine kinase cell signalling pathways which are generally deregulated in $\mathrm{HCC}^{6,7}$ Sorafenib remarkably prolonged the survival rates in advanced HCC patients as illustrated by randomized controlled clinical trials. However, the management of HCC is still debatable. ${ }^{8}$ The reason behind this, is the development of adaptive drug resistance against sorafenib therapy. ${ }^{9} 10 \mathrm{Global}$ analysis of the expressed cellular proteins may aid to identify the differentially expressed proteins between parental cells and drug resistant cancer cells. Besides, malignant cell culture models are also suitable for application of proteomic techniques in order to recognize particular protein that might be associated with a characterized phenotype of the malignant cells."

Recent studies have demonstrated that various alterations in the cytoskeletal proteins may be one of the important mechanisms involved in the development of drug resistance in cancer cells that may in turn be associated with altered drug efflux pumps. ${ }^{12}$ Among them, vimentin is the cytoskeletal protein which belongs to the family of intermediate filament and is one of the markers of epithelial-mesenchymal transition (EMT). ${ }^{13}$ The vimentin expression in cancers is associated with increased tumor invasion and proliferation. Deregulation of vimentin expression and its relation to tumor metastasis has been discussed in various cancers such as gastrointestinal tumors, ${ }^{14,15}$ prostate carcinoma, ${ }^{16,17}$ and breast cancer. ${ }^{18,19} \mathrm{~A}$ relation between vimentin expression and drug resistance has been demonstrated in ovarian cancer as the downregulation of vimentin is associated with acquired drug resistance to cisplatin. ${ }^{20}$ Sorafenib-resistant cancer cells may undergo EMT, however, various studies have shown that sorafenib downregulate this process in $\mathrm{HCC}$ cells. During the exposure of mouse primary hepatocytes to sorafenib, EMT gets diminished due to decrease in transforming growth factor $\beta$ signalling. ${ }^{21} \mathrm{~A}$ study by van Malenstein et al. ${ }^{10}$ in 2013, also have shown that long term exposure of sorafenib to HepG2 cells leads to development of resistance due to activation of EMT. But no such study has yet been revealed EMT cytoskeleton proteins as a potential target for treatment along with sorafenib. Further, the exact role of vimentin is not well established in sorafenib-resistant HCC.

In this study, we have unveiled the expression pattern of vimentin in parental as well as sorafenib-resistant HepG2 cells and further, highlighted the significance of vimentin as a plausible therapeutic target in sorafenib resistant HCC model cell line, HepG2.

\section{MATERIALS AND METHODS}

\section{Cell lines and cell culture}

This study was performed on HepG2 cell line that procured from cell repository of National Centre for Cell Sciences, Pune, India. HepG2 cells were then cultured in minimum essential media (MEM) supplemented with 10\% (v/v) heat-inactivated fetal bovine serum (FBS), $0.15 \%$ (v/v) sodium bicarbonate, $1 \mathrm{mM}$ sodium pyruvate and $2 \mathrm{mM}$ L-glutamine. HepG2 cells were maintained at $37^{\circ} \mathrm{C}$ in $5 \% \mathrm{CO}_{2}$ and $95 \%$ humidity in a $\mathrm{CO}_{2}$ incubator (Thermo Heraeus

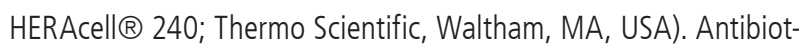
ics streptomycin $(100 \mu \mathrm{g} / \mathrm{mL})$ and penicillin $(100 \mathrm{U} / \mathrm{mL})$ were supplemented in the media for maintenance of the cultures.

\section{Chemicals}

The media and antibiotics for cell culture were procured from HiMedia (Chandigarh, India), Sigma-Aldrich (St. Louis, MO, USA), and ThermoFisher Scientific. Sorafenib was procured from Santacruz Biotechnology, Inc. (Dallas, TX, USA). Withaferin A was procured from Cayman Chemicals Co-USA (Cayman Chemical, Ann Arbor, MI, USA). Both sorafenib and withaferin A were dissolved in dimethyl sulfoxide (DMSO) to prepare $1 \mathrm{mM}$ stock and $20 \mathrm{mM}$ stocks respectively for further use in cell lines.

\section{Samples preparation for matrix-assisted laser desorption/ionization (MALDI)}

The total protein isolated from both HepG2 parental [HepG2 (P)] and HepG2 sorafenib-resistant [HepG2 (R)] cells was estimated following bicinchonic acid method (Sigma-Aldrich). The proteins were separated by sodium dodecyl sulfate polyacrylamide gel 
electrophoresis (SDS-PAGE) according to the method of Laemmeli (1970). After washing the gel with water, the spot of interest was excised and $200 \mu \mathrm{L}$ of distaining solution was added to the gel, vortexed and incubated for 5 minutes and then the supernatant was discarded in order to destain the gel. Gel particles were then treated with $10 \mathrm{mM}$ dithiotreitol/50 mM NH${ }_{4} \mathrm{HCO}_{3}$ (freshly prepared), followed by incubation for 45 minutes at $56^{\circ} \mathrm{C}$ and then immediate cooling of tubes at room temperature. The gel pieces were treated with light sensitive $55 \mathrm{mM}$ idoacetamide prepared in freshly prepared $50 \mathrm{mM} \mathrm{NH}_{4} \mathrm{HCO}_{3}$ for 30 minutes at room temperature and then washed with wash buffer. Freshly prepared trypsin enzyme solution $(30 \mathrm{ng}$ ) was added to the gel, incubated at $37^{\circ} \mathrm{C}$ overnight. Following overnight incubation, the supernatant was collected and $5 \mu \mathrm{L}$ of extraction buffer containing $60 \%$ $\mathrm{ACN}+0.1 \%$ TFA in $25 \mathrm{mM} \mathrm{NH}_{4} \mathrm{HCO}_{3}$ was added to the gel, vortexed and incubated for 10 minutes. Supernatant was collected into the same tube in which supernatant collected from trypsin digestion was added. The above process was then repeated first with extraction buffer containing 70\% ACN+ 0.1 TFA in $25 \mathrm{mM}$ $\mathrm{NH}_{4} \mathrm{HCO}_{3}$ and then with $90 \% \mathrm{ACN}+0.1 \%$ TFA in $25 \mathrm{mM} \mathrm{NH}_{4} \mathrm{H}-$ $\mathrm{CO}_{3}$. The extracted peptides were stored at $-20^{\circ} \mathrm{C}$ for MALDI analysis.

\section{MALDI-time of flight (TOF) mass spectroscopy}

MALDI spectra were acquired using an Ultraflex TOF/TOF mass spectrometer (BrukerDaltonic, Hamburg, Germany). The resulting peaks were subjected to MASCOT search (www.matrixscience. com) and parameters were set as carbamidomethyl modification of cysteine, one missed cleavage was allowed for trypsin (SigmaAldrich). Once protein was identified it was further fragmented and subjected to MS-MS analysis. Protein MASCOT score, sequence coverage, and number of peptides matched were used to confirm the protein using SwissProt database (www.uniprot.org).

\section{RNA isolation, CDNA synthesis and quantitative real- time polymerase chain reaction (qRT PCR) analysis}

Total RNA was isolated from both HepG2 (P) as well as HepG2 (R) cells using the Trizol reagent (Invitrogen, Carlsbad, CA, USA). Finally, RNA concentration was quantified and processed for CDNA synthesis. CDNA synthesis was carried out from the intact RNA by using Verso CDNA Synthesis Kit (Thermofischer). qRT PCR was performed on Lightcycler ${ }^{\circledR} 96$ (Roche, Bremen, Germany) using SYBR Green I master (Roche) detection method to check relative expression of target genes using specific primers for glyceraldehyde 3-phosphate dehydrogenase (GAPDH), vimentin and $A B C G 2$ genes. The relative mRNA levels were calculated using the formula $2^{-\Delta \Delta \mathrm{Ct}}$, where $\Delta \mathrm{Ct}=(\mathrm{Ct}$ target gene - $\mathrm{Ct}$ internal control). Each set of primers were designed to target specifically exon-exon junction in CDNA and was commercially synthesized by Sigma-Aldrich, USA. The gene expression was normalized using GAPDH acting as internal control. The primer sequences are:

ABCG2 (F)-GTGGCCTTGGCTTGTATGAT (R)-GATGGCAAGGGAACAGAAAA

GAPDH (F)-CCATCTTCCAGGAGCGAGA (R)-GGTCATGAGTCCTTCCACGAT

Vimentin (F)-CCGGTGCAATCGTGATCTCTGGG (R)-ATTCAAGTCTCAGCGGGCTC

\section{Protein expression: flow cytometry}

HepG2 (P) and HepG2 (R) cells were cultured in a 12-well plate. After 24 hours of treatment, cells were trypsinized and washed twice with phosphate-buffered saline (PBS) in dark and cell pellet was resuspended in PBS. The cells were then fixed using $4 \%$ paraformaldehyde solution and again washed using PBS. After washing, the cells were permeabilized using permeabilizing buffer (1\% BSA $+0.01 \%$ Triton X $100+0.01 \%$ Sodium azide in $1 X$ PBS) and washed with PBS. Then blocking buffer (5\% BSA in 1X PBS) was used to blocking the cells. Antibody solution was added after blocking to cells and incubated for 1 hour at room temperature. The expression of vimentin was then analyzed using flow cytometry.

\section{MTT assay}

HepG2 (P) and HepG2 (R) cells were seeded in a 96-wells plate (Corning Inc, Corning, NY, USA) with density of 5,000 cells per well in MEM with 10\% FBS. After 24 hours of incubation, various concentrations (conc.) of withaferin A and sorafenib were added to MEM with $0.5 \% \mathrm{FBS}$, a total volume of $200 \mu \mathrm{L}$ per well. After 24 hours, sterile $20 \mu \mathrm{L}$ MTT solution was added to each well and plates were incubated for 4 hours at $37^{\circ} \mathrm{C}, 5 \% \mathrm{CO}_{2}$. Media containing MTT was then removed from the each well and formazan crystals formed were dissolved in DMSO $(200 \mu \mathrm{L})$ and incubated for 5 to 15 minutes at room temperature in dark. The absorbance was then measured at $570 \mathrm{~nm}$ using a microtiter plate reader by infinite $_{\text {M200pro }}$ microplate reader (Tecan, Männedorf, Switzerland). 


\section{Crystal violet assay}

This analysis was done to assess the growth inhibition pattern of HepG2 (P) and HepG2 (R) cells under different conditions. HepG2 cells (2,000 per well) were seeded in a 24-well plate in triplicate. After 24 hours of treatment, the cells were washed with PBS (1X) and later fixed using 4\% paraformaldehyde in PBS (1X) for 15 minutes (200 $\mu \mathrm{L}$ per 12-well). Then, the cells were stained with $0.1 \%$ crystal violet $(50 \mathrm{mg}$ crystal violet powder in $5 \mathrm{~mL}$ ethanol/45 mL water) for 20 minutes (100 $\mu \mathrm{L}$ per 12-well). Subsequently, after washing with PBS, $500 \mu \mathrm{L} 10 \%$ acetic acid was added to each well and incubated for 20 minutes on shaker. Finally, out $0.5-1 \mathrm{~mL}$ of stain was taken out and absorbance was checked at $590 \mathrm{~nm}$.

\section{Statistical analysis}

For the statistical analysis the experimental group differences were calculated by Student's $t$-test or one-way ANOVA with Bonferroni's correction for multiple comparisons. Drug concentrations effect in two cell types were assessed by two-way ANOVA with Sidak's correction. All data were analyzed by GraphPad Prism (v.4.00; GraphPad Software, San Diego, CA, USA). Data were presented as the mean and standard error of the mean. Significance level was set at 0.05 .

\section{RESULTS}

Stored HepG2 (P) and HepG2 (R) cells were recultured to perform the present experiments. As earlier (Ankita Makol, Ph.D. thesis, 2017) the HepG2 cell line was treated with minimal concentrations of sorafenib inhibitory concentration (IC)-20 dissolved in DMSO, continuously for long period followed by further fractional dose elevation to mimic the clinical settings. As a result, sorafenib-resistant HepG2 cell line was established. ${ }^{22}$ The chemoresistance nature of HepG2 cells was characterized in view of microscopic analysis (Fig. 1A) and dose-response assessment (IC-50) in presence of different doses of the sorafenib (Fig. 1B). The IC-50 value of resistant cells was 2.25 fold higher than parental cells.

\section{Protein profiling and identification of differentially expressed proteins in HepG2 cells}

In order to detect candidate protein marker which may have role in development of resistance against sorafenib, the comparative protein profiling of HepG2 (P) and HepG2 (R) cells was done using SDS-PAGE followed by coomassie staining (Fig. 2A). The six fragments $(1,2,3,4,5$, and 6$)$ on the coomassie stained gel (Fig. 2B) were selected for MALDI-TOF-MS analysis. Among them peptide mass fingerprinting (PMF) of each in-gel digested four samples along with the respective theoretical weight, protein coverage, peptide matches which were documented from Swiss-Prot for each samples (Supplementary Table 1). The identified proteins were further confirmed by MS analysis. The analysis resulted in the identification of four proteins which were differentially expressed in HepG2 (P) and HepG2 (R) cells.

According to PMF analysis, sample 1 (excised protein band) was identified to be glucose regulated protein 78 (GRP78) which is highly expressed in HepG2 (R) as compared to HepG2 (P) cells whereas sample 2 was identified to be actin, highly expressed in HepG2 (R) in comparison to HepG2 (P) cells. On the contrary,

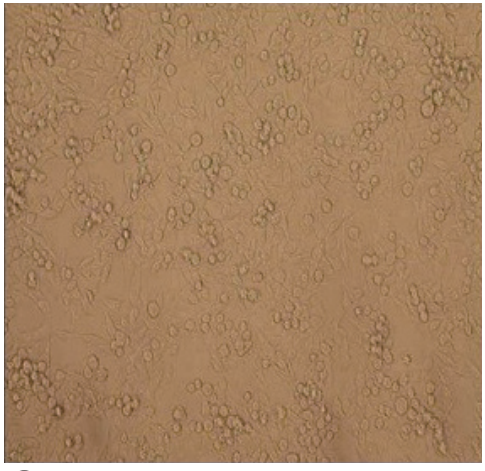

A $\quad \operatorname{HepG2}(\mathrm{P})$

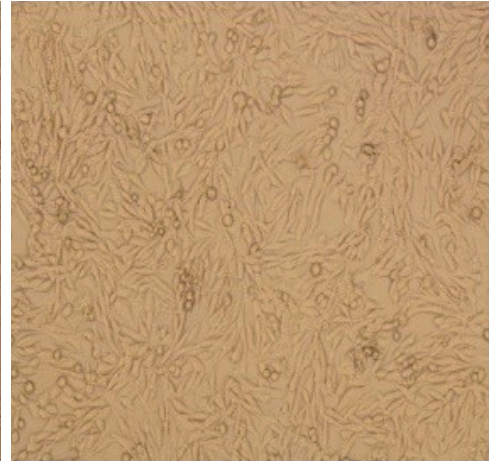

$\operatorname{HepG2}(\mathrm{R})$

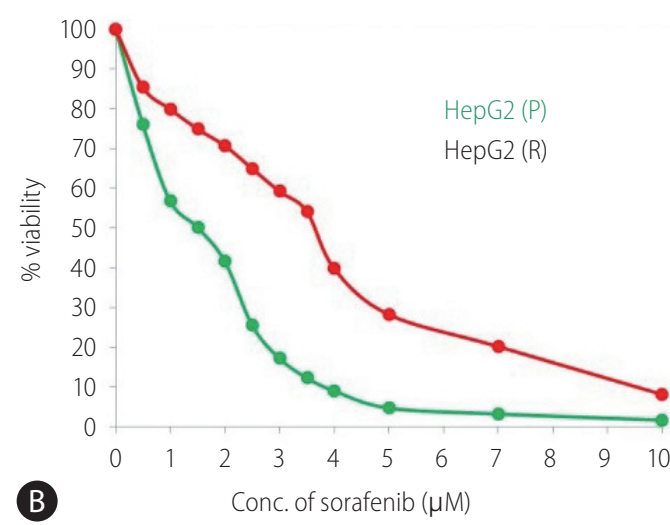

Figure 1. (A) Morphological analysis of HepG2 (P) and HepG2 (R) cells. (B) Sorafenib dose response curve [IC50 HepG2 (P) = $1.6 \mu \mathrm{M}$; IC50 HepG2 (R) = 3.6 M; fold resistance = 2.25]. HepG2 (P), HepG2 parental; HepG2 (R), HepG2 sorafenib-resistant; Conc., concentrations. 

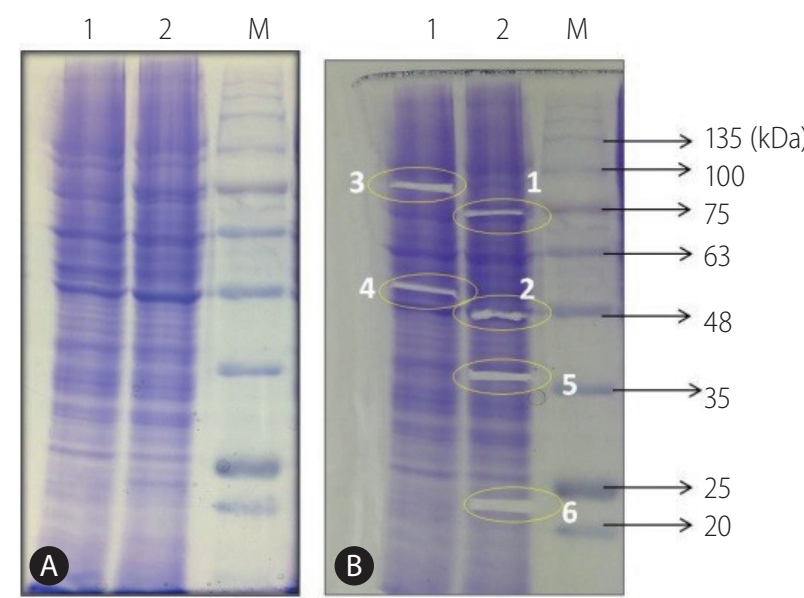

Figure 2. (A) Protein profile of HepG2 (P) and (R) cells on 10\% SDS-PAGE stained with Coomassie brilliant blue. (B) Excision of protein bands from HepG2 cells [lanes: 1-HepG2 (P); 2-HepG2 (R); M-BLUelf Prestained Protein Ladder]. HepG2 (P), HepG2 parental; HepG2 (R), HepG2 sorafenib-resistant; SDS-PAGE, sodium dodecyl sulfate polyacrylamide gel electrophoresis. sample 3 was identified to be TRAP1 that was highly expressed in HepG2 (P) cells as compared to HepG2 (R) cells. Sample 4 was identified to be vimentin with theoretical mass $50 \mathrm{kDa}$ which was similar to observed molecular weight $(53,676 \mathrm{Da})$, protein coverage $44 \%$, peptide matches 31 . Vimentin was further confirmed by MS-MS analysis. Two peptides of molecular weight 1,572 Da and 1,094 Da were analysed which showed the identification with peptides of vimentin which was highly expressed in HepG2 (P) cells in comparison to HepG2 (R) cells (Supplementary Fig. 1).

\section{Expression of vimentin in HepG2 (P) and HepG2 (R) cells}

The expression level of vimentin was examined in both HepG2 (P) and HepG2 (R) cells at mRNA levels by qRT-PCR. Vimentin gene expression was resulted to be downregulated in HepG2 (R) cells in comparison to HepG2 (P) (Fig. 3C). These results were further confirmed at protein level through analysis of vimentin by flow cytometry. The protein expression analysis using phycoery-

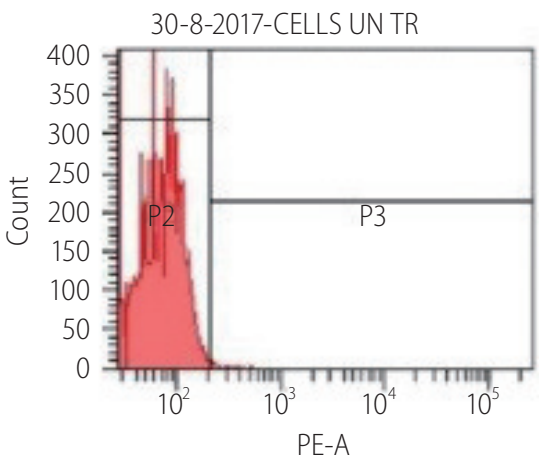

(i)

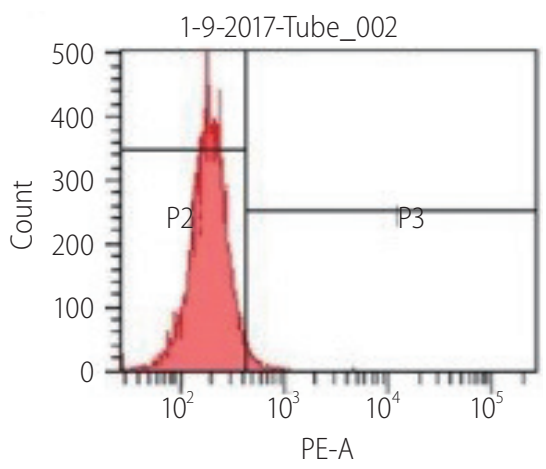

A

(iv)

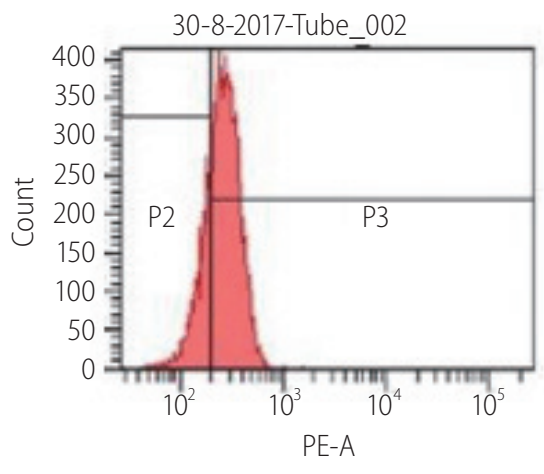

(ii)

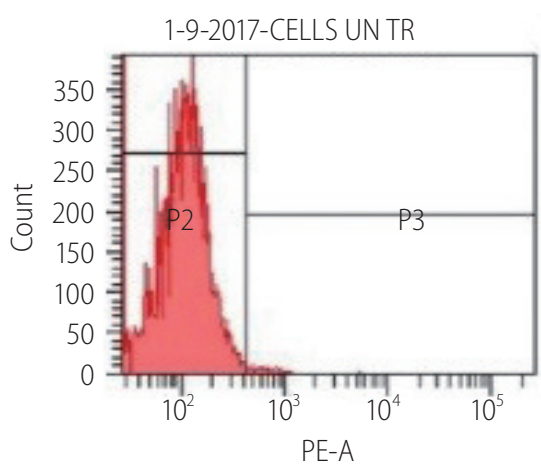

(iii)

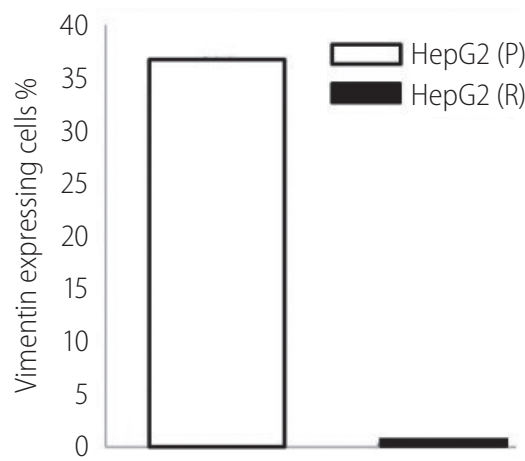

B

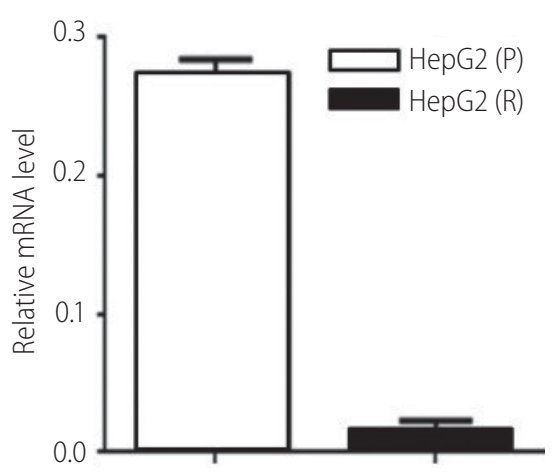

C

Figure 3. (A) Representative FACS plots of: (i) HepG2 (P) cells without antibody (control), (ii) HepG2 (P) cells with anti-vimentin antibody, (iii) HepG2 (R) cells without antibody (control), and (iv) HepG2 (R) cells with anti-vimentin antibody. (B) Protein level expression of vimentin in HepG2 (P) and HepG2 (R) cells. (C) Vimentin expression in HepG2 (P) and HepG2 (R) cells. HepG2 (P), HepG2 parental; HepG2 (R), HepG2 sorafenib-resistant; FACS, fluorescence-activated cell sorting. 
therin labelled anti-vimentin antibodies (mouse anti-human antibodies) revealed higher expression of vimentin in HepG2 (P) cells as compared to HepG2 (R) cells (Fig. 3A, B).

\section{Vimentin expression in response to sorafenib}

In the presence, of different concentrations of sorafenib (1.0, $2.5,5.0$, and $7.5 \mu \mathrm{M})$, the relative expression levels of vimentin in HepG2 (P) and HepG2 (R) cells has shown deregulated behaviour. Moreover, at sorafenib conc. $5 \mu \mathrm{M}$, vimentin expression was significantly decreased in HepG2 (R) cells. Therefore, this concentration was selected for further experiments (Fig. 4).

\section{Vimentin expression in relation to withaferin $A$ and its combination with sorafenib}

Withaferin $A$ is a steroidal lactone found in the restorative plant, Withania somnifera (Ashwagandha). It is reported as a potent inhibitor of vimentin, as it is known to cause the aggregation of tetrameric form of vimentin. ${ }^{23}$ We found that gene expression levels of vimentin was downregulated in the HepG2 (R) cells when treated with increasing concentrations of withaferin A. In HepG2 (R) cells, the vimentin expression was significantly decreased at withaferin A drug concentration of $0.25 \mu \mathrm{M}$ (IC-50 value; $>6$ fold decrease).

Further, we also examined the gene expression of vimentin in HepG2 (R) cells in response to the combination of withaferin A and sorafenib as compared to the untreated ones. It was observed

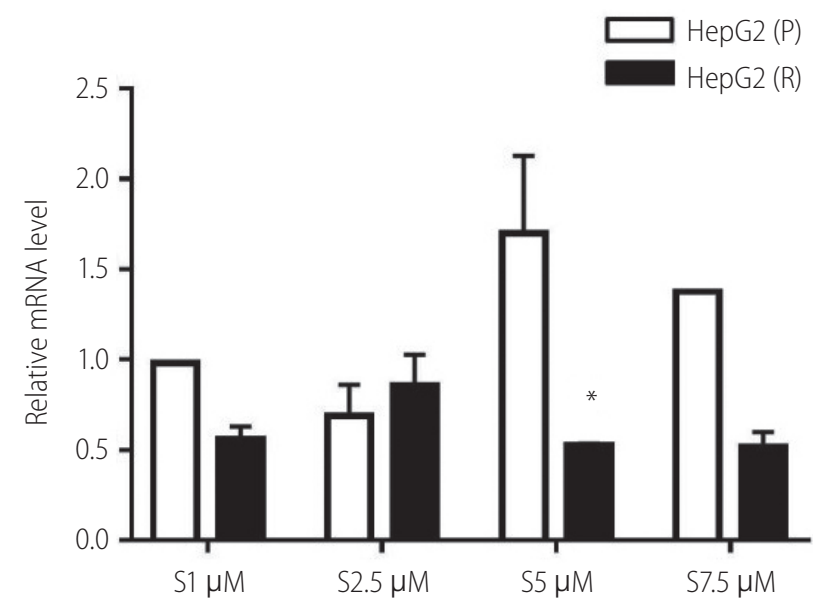

Figure 4. Vimentin expression in response to different concentrations of sorafenib (S) in HepG2 cells. HepG2 (P), HepG2 parental; HepG2 (R), HepG2 sorafenib-resistant. ${ }^{*} P=0.263$. that when HepG2 (R) cells were treated with the combination of sorafenib $(5 \mu \mathrm{M})$ and withaferin $\mathrm{A}(0.25 \mu \mathrm{M}$; IC-50 value), the gene expression of vimentin showed a significant decrease in comparison to untreated cells (Fig. 5).

\section{Effect of vimentin inhibition on sorafenib resistance: expression analysis of $A B C G 2$}

$A B C G 2$ transporters are known to be commonly associated with the development of drug resistance in majority of cancers. ${ }^{24}$ Elucidating the targeted effect of withaferin $\mathrm{A}$ on the $A B C G 2$ gene expression in HCC cells, we analyzed the effects of its incremented doses as well as in combination with sorafenib.

As expected, the $A B C G 2$ gene expression was higher in HepG2 (R) cells as compared to HepG2 (P) cells (Fig. 6A). Further, the levels of $A B C G 2$ gene were observed to decrease with $0.25 \mu \mathrm{M}$ (IC50 value) withaferin $A$ treatment in $\operatorname{HepG2}(R)$ cells and at drug concentrations of $0.5 \mu \mathrm{M}$, the levels of $A B C G 2$ were observed to decrease significantly (Fig. 6B).

\section{Effect of vimentin inhibition on cell viability of HepG2 cells}

The effect on cell viability via withaferin A treatment and combination of withaferin A with sorafenib was assayed through crystal violet staining. This analysis depicted the dose dependent cytotoxic effect of withaferin $A$ and combination of withaferin $A$ with sorafenib. The cell number significantly decreased with increasing concentration of withaferin $A$ as well as with the combination of sorafenib and withaferin A (Fig. 7A).

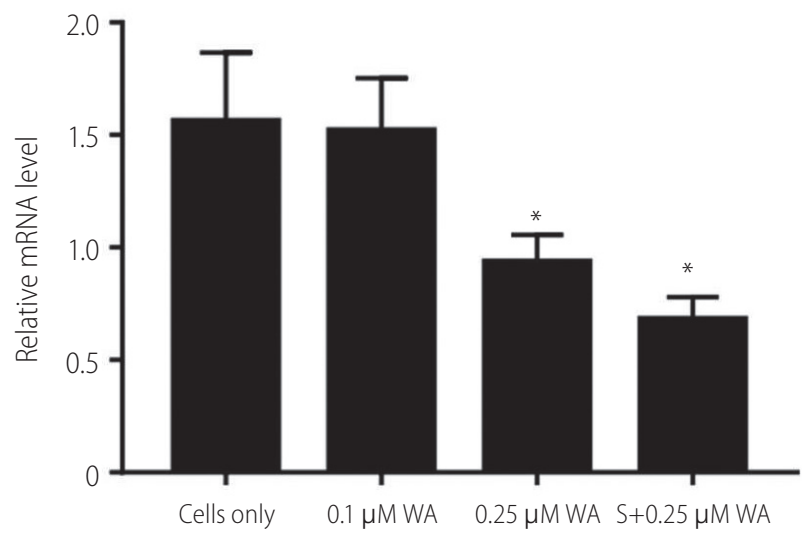

Figure 5. Vimentin expression in HepG2 (R) cells in response to: withaferin $\mathrm{A}(\mathrm{WA})$ and a combination of sorafenib $(S ; 5 \mu \mathrm{M})$ and WA. HepG2 (R), HepG2 sorafenib-resistant. ${ }^{*} P=0.034$. 
The cell viability of HepG2 (P) and HepG2 (R) cells was also confirmed through the MTT assay. On treating the cells with different concentrations of withaferin $\mathrm{A}$ as well as in combination with sorafenib $(5 \mu \mathrm{M})$, the cell viability was found to decrease significantly in both HepG2 (P) and HepG2 (R) cells (Fig. 7B).

As increasing concentration of withaferin $A$ as well as its combination with sorafenib, the only first-line therapy for advanced HCC has a direct effect on cell viability of both HepG2 (P) and HepG2 (R) cells.

Hence, inhibition of vimentin might involve modulating sorafenib resistance as well as proliferation of HCC.

\section{DISCUSSION}

$\mathrm{HCC}$ is the primary cancer of liver and the leading cause of death in patients with liver diseases. The first-line systemic therapy of choice for advanced HCC is sorafenib that is known to extend the median survival time moderately by $2-3$ months. However, long-term exposure of sorafenib has been reported to induce adaptive resistance in patients. The main processes which are known to have a role in sorafenib resistance broadly include hypoxic microenvironment, autophagy and EMT etc. EMT is a process of acquiring more metastatic and invasive properties similar to mesenchymal cells and is stated as losing the normal epithelial properties, like cellular polarity and cell-cell contact by the epithelial cells. Vimentin, a cytoskeleton protein of $57 \mathrm{kDa}$, is a highly conserved and broadly expressed protein of the type III Intermediate Filament protein family. Vimentin expression is restricted to mesenchymal cells. Vimentin has gained much attention in cancer biology as a sanctioned marker of EMT, lately. EMT is a cellular reprogramming process in which the epithelial cells lose their cellu-
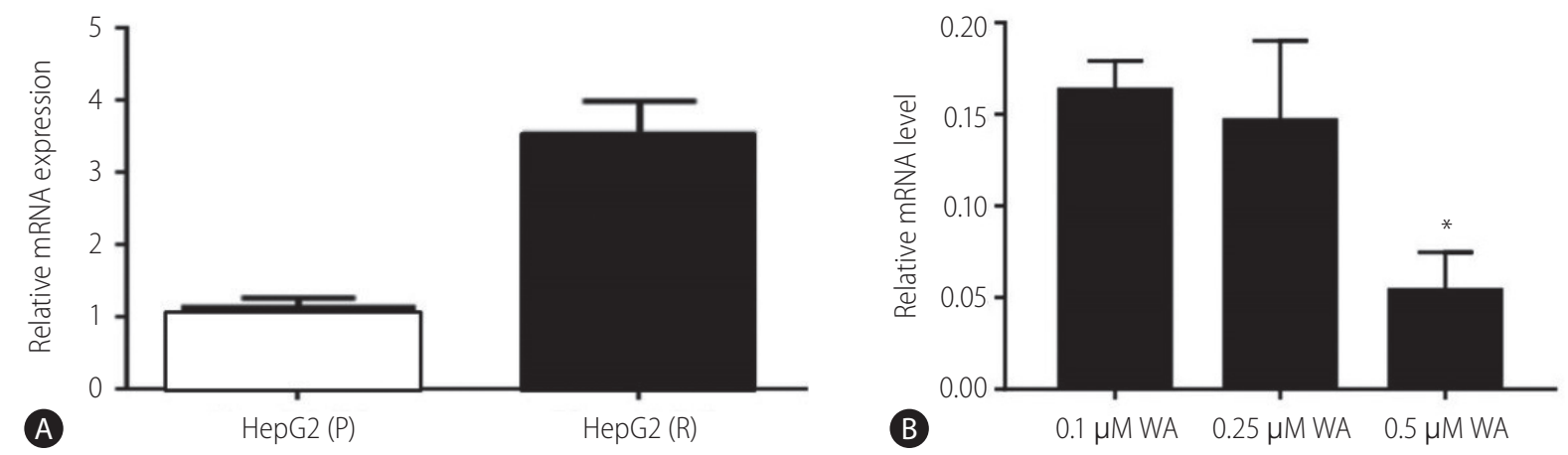

Figure 6. Effect of vimentin inhibition on sorafenib resistance: (A) ABCG2 expression in HepG2 cells. (B) In response to withaferin $A(W A)$ in HepG2 (R) cells. HepG2 (P), HepG2 parental; HepG2 (R), HepG2 sorafenib-resistant. ${ }^{*} P=0.021$.

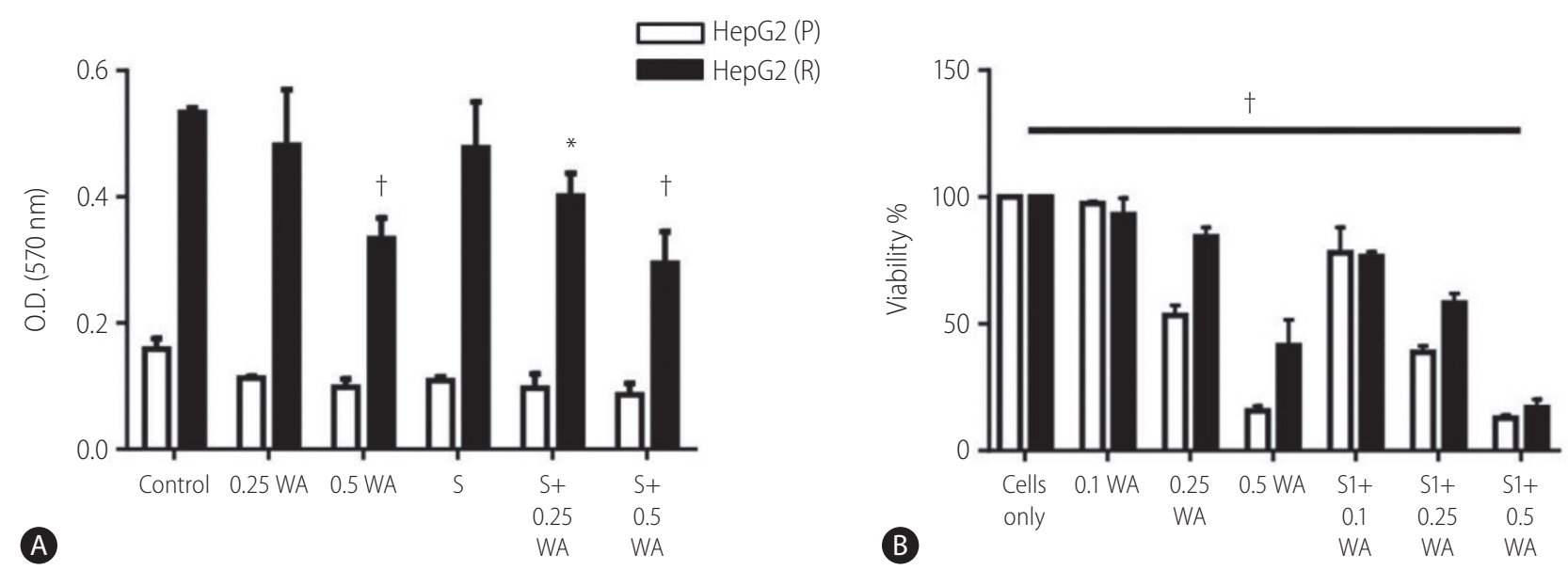

Figure 7. Effect of vimentin inhibition with withaferin $A(W A)$ and WA in combination with sorafenib $(S)$ on the overall cell viability of HepG2 cells via: (A) crystal violet staining and (B) MTT assay. HepG2 (P), HepG2 parental; HepG2 (R), HepG2 sorafenib-resistant. ${ }^{*} P=0.0003,{ }^{\dagger} P<0.0001$. 
lar polarity, downregulation of the epithelial markers like E-cadherins and keratin, and acquires a mesenchymal phenotype that results in change of shape and shows increased motility of cells. The process of EMT is related to the development of resistance against certain chemotherapeutic drugs and vimentin expression has been observed to be upregulated in various tumor cell lines and tissues. Thus, we would like to study the role of vimentin in proliferation as well as sorafenib resistance in hepatocellular carcinoma.

In present study, the proteomic profiling of parental [HepG2 (P)] and sorafenib-resistant [HepG2 (R)] cells revealed the downregulation of vimentin in HepG2 (R) cells as compared to HepG2 (P) cells. This result was further confirmed at mRNA level by qRT PCR and at protein level by flow cytometry analysis. A study in 2016 by Yi Huo et al. also reported the similar pattern of downregulation of vimentin in cisplatin resistant ovarian cancer cells. Then, we investigated the effect of different doses of sorafenib on gene expression of vimentin and at $5 \mu \mathrm{M}$ sorafenib conc. there is significant downregulation of vimentin expression. Further, to evaluate significance of vimentin inhibition in sorafenib resistance, a well known inhibitor of vimentin was used that mediates its aggregation and disassembly. Withaferin A successfully lowered down the levels of vimentin in HepG2 (R) cells.

In HCC sorafenib resistance, $A B C G 2$ is one of the prominent efflux pumps belonging to the family of ABC (ATP binding cassette) transporters, involved in efflux of sorafenib out of resistant cancer cells..$^{25,26}$ We analyzed the effect of withaferin A on $A B C G 2$ expression in HepG2 (R) cells to understand its targeted action. In our study, withaferin A significantly decreased the levels of ABCG2 gene in HepG2 (R) cells which indicated towards the probable role of vimentin in the establishment of sorafenib resistance in HCC cells.

Studies reported about the cytotoxic effect of Withaferin A in cancer cells. ${ }^{27}$ To evaluate its cytotoxic nature our both crystal violet as well as MTT assays has demonstrated that using increasing dose of withaferin $A$ as well as in combination with sorafenib both resulted to decrease cell viability of both HepG2 (P) and HepG2 (R) cells significantly.

Thus, the modulation of vimentin may result in the shift in proliferation as well as sorafenib resistance in HCC. Hence, our results demonstrated that vimentin is an eminent marker as well as its significance as a potential therapeutic target to treat sorafenib resistance in HCC.

This study connotes that vimentin is crucial for cell survival as its inhibition resulted in cell cytotoxicity in both HepG2 (P) and
HepG2 (R) cells. Nonetheless, vimentin expression in resistant HCC cells was observed to be lowered, the inhibition further has proved detrimental to the resistant cells. Further, deciphering the specific resistance mediators, the effect of withaferin A was checked on $A B C$ transporter gene. For the first time, the present study suggesting that withaferin $A$ leads to a decrease in the expression of $A B C G 2$ gene in sorafenib-resistant HCC [HepG2 (R)] cells. The inhibitor, withaferin $A$, mediating its anti-cancer action through cell apoptosis significantly decreased the expression of vimentin as well as cell viability in a dose dependent manner. This observed shift in resistant cells towards a parental-cell profile further hints the effect of vimentin in sorafenib resistance as its inhibition with withaferin $A$ as a combination therapy along with only current targeted drug, sorafenib.

However, our study has some limitations. All experiments were performed using only one human HCC cell line (HepG2) and it was not possible to perform in vivo experiments. Thus, future research in this area is required to completely understand the mechanism and validate this study.

\section{Authors' contribution}

AM, HK, SS, SK, RK were participated in the study concept and its design, acquisition of data, analysis and interpretation of data, and drafting of the manuscript. Anuradha Chakraborti participated in study supervision, data interpretation, and critical review of the manuscript for important intellectual content.

\section{Acknowledgements}

We acknowledge Indian Council of Medical Research (ICMR) for providing senior research fellowship to Ankita Makol, Shruthi Kanthaje and Raman Kaur.

\section{Conflicts of Interest}

The authors have no conflicts to disclose.

\section{SUPPLEMENTARY MATERIALS}

Supplementary materials are available at Clinical and Molecular Hepatology website (http://www.e-cmh.org).

\section{REFERENCES}

1. Badvie S. Hepatocellular carcinoma. Postgrad Med J 2000;76:4-11. 
2. Llovet JM, Zucman-Rossi J, Pikarsky E, Sangro B, Schwartz M, Sherman $M$, et al. Hepatocellular carcinoma. Nat Rev Dis Primers 2016;2:16018.

3. Feitelson MA, Sun B, Satiroglu Tufan NL, Liu J, Pan J, Lian Z. Genetic mechanisms of hepatocarcinogenesis. Oncogene 2002;21:25932604.

4. Murakami Y, Saigo K, Takashima H, Minami M, Okanoue T, Bréchot C, et al. Large scaled analysis of hepatitis B virus (HBV) DNA integration in HBV related hepatocellular carcinomas. Gut 2005;54:11621168.

5. Tinkle CL, Haas-Kogan D. Hepatocellular carcinoma: natural history, current management, and emerging tools. Biologics 2012;6:207219.

6. Adnane L, Trail PA, Taylor I, Wilhelm SM. Sorafenib (BAY 43-9006, Nexavar), a dual-action inhibitor that targets RAF/MEK/ERK pathway in tumor cells and tyrosine kinases VEGFR/PDGFR in tumor vasculature. Methods Enzymol 2006;407:597-612.

7. Wilhelm SM, Carter C, Tang L, Wilkie D, McNabola A, Rong H, et al. BAY 43-9006 exhibits broad spectrum oral antitumor activity and targets the RAF/MEK/ERK pathway and receptor tyrosine kinases involved in tumor progression and angiogenesis. Cancer Res 2004;64:7099-7109.

8. Cheng AL, Kang YK, Chen Z, Tsao CJ, Qin S, Kim JS, et al. Efficacy and safety of sorafenib in patients in the Asia-Pacific region with advanced hepatocellular carcinoma: a phase III randomised, doubleblind, placebo-controlled trial. Lancet Oncol 2009;10:25-34.

9. Wada Y, Takami Y, Tateishi M, Ryu T, Mikagi K, Saitsu H. The efficacy of continued sorafenib treatment after radiologic confirmation of progressive disease in patients with advanced hepatocellular carcinoma. PLoS One 2016;11:e0146456.

10. van Malenstein H, Dekervel J, Verslype C, Van Cutsem E, Windmolders $P$, Nevens $F$, et al. Long-term exposure to sorafenib of liver cancer cells induces resistance with epithelial-to-mesenchymal transition, increased invasion and risk of rebound growth. Cancer Lett 2013;329:74-83.

11. Lage H. Proteomics in cancer cell research: an analysis of therapy resistance. Pathol Res Pract 2004;200:105-117.

12. Kanakkanthara A, Teesdale-Spittle PH, Miller JH. Cytoskeletal alterations that confer resistance to anti-tubulin chemotherapeutics. Anticancer Agents Med Chem 2013;13:147-158.

13. Thiery JP. Epithelial-mesenchymal transitions in tumour progression. Nat Rev Cancer 2002;2:442-454.

14. Fuyuhiro Y, Yashiro M, Noda S, Kashiwagi S, Matsuoka J, Doi Y, et al. Clinical significance of vimentin-positive gastric cancer cells. Anticancer Res 2010;30:5239-5243.
15. Takemura K, Hirayama R, Hirokawa K, Inagaki M, Tsujimura K, Esaki $Y$, et al. Expression of vimentin in gastric cancer: a possible indicator for prognosis. Pathobiology 1994;62:149-154.

16. Zhao Y, Yan Q, Long X, Chen X, Wang Y. Vimentin affects the mobility and invasiveness of prostate cancer cells. Cell Biochem Funct 2008;26:571-577.

17. Lang SH, Hyde C, Reid IN, Hitchcock IS, Hart CA, Bryden AA, et al. Enhanced expression of vimentin in motile prostate cell lines and in poorly differentiated and metastatic prostate carcinoma. Prostate 2002;52:253-263.

18. Gilles C, Polette M, Mestdagt M, Nawrocki-Raby B, Ruggeri P, Birembaut $P$, et al. Transactivation of vimentin by beta-catenin in human breast cancer cells. Cancer Res 2003;63:2658-2664.

19. Korsching E, Packeisen J, Liedtke C, Hungermann D, Wulfing P, van Diest PJ, et al. The origin of vimentin expression in invasive breast cancer: epithelial-mesenchymal transition, myoepithelial histogenesis or histogenesis from progenitor cells with bilinear differentiation potential? J Pathol 2005;206:451-457.

20. Kanakkanthara A, Rawson P, Northcote PT, Miller JH. Acquired resistance to peloruside $A$ and laulimalide is associated with downregulation of vimentin in human ovarian carcinoma cells. Pharm Res 2012;29:3022-3032.

21. Chen YL, Lv J, Ye XL, Sun MY, Xu Q, Liu CH, et al. Sorafenib inhibits transforming growth factor $\beta 1$-mediated epithelial-mesenchymal transition and apoptosis in mouse hepatocytes. Hepatology 2011;53:1708-1718.

22. Zhang X, Yashiro M, Qiu H, Nishii T, Matsuzaki T, Hirakawa K. Establishment and characterization of multidrug-resistant gastric cancer cell lines. Anticancer Res 2010;30:915-921.

23. Lee J, Hahm ER, Marcus Al, Singh SV. Withaferin A inhibits experimental epithelial-mesenchymal transition in MCF-10A cells and suppresses vimentin protein level in vivo in breast tumors. Mol Carcinog 2015;54:417-429.

24. Mo W, Zhang JT. Human ABCG2: structure, function, and its role in multidrug resistance. Int J Biochem Mol Biol 2012;3:1-27.

25. Huang WC, Hsieh YL, Hung CM, Chien PH, Chien YF, Chen LC, et al. BCRP/ABCG2 inhibition sensitizes hepatocellular carcinoma cells to sorafenib. PLoS One 2013;8:e83627.

26. Chen YL, Chen PM, Lin PY, Hsiau YT, Chu PY. ABCG2 overexpression confers poor outcomes in hepatocellular carcinoma of elderly patients. Anticancer Res 2016;36:2983-2988.

27. Thaiparambil JT, Bender L, Ganesh T, Kline E, Patel P, Liu Y, et al. Withaferin $A$ inhibits breast cancer invasion and metastasis at subcytotoxic doses by inducing vimentin disassembly and serine 56 phosphorylation. Int J Cancer 2011;129:2744-2755. 